\title{
A Functional Assay for Putative Mouse and Human Definitive Endoderm using Chick Whole-Embryo Cultures
}

\author{
Martina Johannesson ${ }^{1}$, Henrik Semb², Palle Serup² and Mattias Hansson ${ }^{1 *}$ \\ ${ }^{1}$ Department of Stem Cell Biology, Hagedorn Research Institute, Niels Steensensvej 6, DK-2820 Gentofte, Denmark \\ ${ }^{2}$ Danish Stem Cell Center, Copenhagen University, Blegdamsvej 3B, DK-2200 Copenhagen, Denmark
}

\begin{abstract}
Introduction: Embryonic stem cells (ESCs) represent a prospective cell source for treating degenerative diseases such as diabetes. Several studies have addressed the generation of definitive endoderm (DE) from this cell source by attempting to recapitulate the signaling events occurring during embryogenesis. However, the subsequent differentiation of DE has failed to generate functional insulin-producing beta cells. To assure that we have the correct starting material, we need to fully characterize ESC-derived DE, by assessing whether the cells are functionally equivalent to the in vivo counterpart. Thus, the purpose of this study is to describe a method whereby the in vivo functionality of DE derived from ESCs can be assessed.
\end{abstract}

Methods: By directed differentiation, putative DE was derived from human and mouse ESCs. This putative DE was subsequently transplanted into the endoderm of chick embryos to determine any occurrence of integration. Putative DE was analyzed by gene and protein expression prior to transplantation and $48 \mathrm{~h}$ post transplantation.

Results: Putative DE, derived from mouse and human ESCs, was successfully integrated within the chick endoderm. Endoderm-specific genes were expressed in the putative DE prior to integration and endoderm-specific proteins were assessed $48 \mathrm{~h}$ post transplantation.

Conclusions: We describe the detailed methodological procedure for transplanting putative DE derived from ESCs, and the subsequent analysis of the migration and development of the grafted cells. Our result show that putative DE integrates with the chick endoderm and participate in the development of the chicken gut, indicating the generation of functional DE from ESCs. This functional assay can be used to assess the generation of functional DE derived from both human and mouse ESCs and provides a valuable tool for cell characterization. This is an important initial step in the differentiation process towards fully functional beta cells.

Keywords: Functional assay; Whole mount immunofluorescence; Embryonic stem cell; Chicken; Definitive endoderm; Diabetes mellitus

Abbreviations: DE: Definitive Endoderm; hDE: human Definitive Endoderm; mDE: mouse Definitive Endoderm; ESC: Embryonic Stem Cells; mESC: mouse Embryonic Stem Cells; hESC: human Embryonic Stem Cells; HH: Hamburger Hamilton; HKG: House-keeping Gene; VE: Visceral Endoderm; PS: Primitive Streak; EMT: Epithelial to Mesenchymal Transition

\section{Introduction}

The transplantation of allogenic islets of Langerhans from cadaveric donors to patients with type 1 diabetes mellitus demonstrates the use of cell replacement therapy in diabetes treatment [1]. Given the lack of donor islets, embryonic stem cells (ESCs) represent a potentially unlimited alternative source of beta cells [2,3]. All gastrointestinal organs, including the pancreas, are derived from the definitive endoderm (DE), one of three germ layers formed during gastrulation [4]. Recent studies have addressed the generation of DE and its subsequent differentiation to pancreatic progenitors and insulinproducing beta-like cells from ESCs by recapitulating the progressive developmental process in vitro [5-7]. Although the endpoint cells derived in vitro are distinctly different from fully functional beta cells, glucose-responsive beta-like cells can be generated by in vivo maturation of ESC-derived progenitors [8]. Importantly, DE cells derived from ESCs must be competent to respond to subsequent in vivo signals, which specify differentiation towards a pancreatic fate. Thus, to fully characterize ESC-derived DE, there is a requirement for a functional assay to analyze its potential [9]. The chick embryo represents a well-characterized, easily accessible, and inexpensive in vivo model $[10,11]$, which has frequently been used to study mammalian development. It has been used to assess the proliferation, differentiation, migration capacity, and/or function of rodent and human undifferentiated and differentiated ESCs [12-18]. Previously, we have reported the integration and differentiation of mouse ESCderived DE after grafting to the endoderm of developing chick embryos [19]. Here, we extend previous findings and present a functional assay where putative DE, derived from mouse ESCs (mESC) or human ESCs (hESC), is grafted to the endoderm of the chick embryo. We describe a detailed methodological procedure for transplanting the putative DE into chick endoderm, followed by whole-mount immunocytochemical analysis of the grafted embryos, to monitor the migration, integration, and development of the grafted cells. In addition, the putative DE is analyzed in vitro by immunocytochemistry and quantitative PCR prior to grafting in order to investigate the phenotype of the cells.

*Corresponding author: Mattias Hansson, Hagedorn Research Institute, Niels Steensens Vej 6, DK-2820 Gentofte, Denmark, Tel: +45 4442 9137; E-mail: mshs@hagedorn.dk

Received July 20, 2012; Accepted September 12, 2012; Published September 14,2012

Citation: Johannesson M, Semb H, Serup P, Hansson M (2012) A Functional Assay for Putative Mouse and Human Definitive Endoderm using Chick WholeEmbryo Cultures. J Stem Cell Res Ther 2:128. doi:10.4172/2157-7633.1000128

Copyright: (c) 2012 Johannesson M, et al. This is an open-access article distributed under the terms of the Creative Commons Attribution License, which permits unrestricted use, distribution, and reproduction in any medium, provided the original author and source are credited. 


\section{Methods}

\section{Differentiation into mouse definitive endoderm}

E14 or Sox17Gfp/+ mESCs were cultured on gelatin-coated (SigmaAldrich) cell culture flasks (Nunc) in serum-free media containing KODMEM supplemented with N2, B27, $0.1 \mathrm{mM}$ non-essential amino acids, $2 \mathrm{mM}$ L-glutamine, Penicillin/Streptomycin (PEST) (all from Gibco/ Invitrogen), $0.1 \mathrm{mM}$ 2-mercaptoethanol (Sigma-Aldrich), $1500 \mathrm{U} /$ $\mathrm{mL}$ leukemia inhibitory factor (LIF, Chemicon), and $10 \mathrm{ng} / \mathrm{mL}$ BMP4 (R\&D Systems), essentially as described by Ying et al. [20]. mESCs were grown in this medium for three passages prior to differentiation, which was induced by seeding $2,000 \mathrm{mESCs} / \mathrm{cm}^{2}$ on gelatin-coated cell culture plastic in the media previously described but without LIF and BMP4. $100 \mathrm{ng} / \mathrm{mL}$ Activin A (R\&D Systems) was added to the media to induce formation of DE from mESCs. The cells were cultured for five days and the medium was changed daily, beginning at the second day of differentiation. Sox17GFP/+ mESCs was obtained from S. Morrison, Department of Cell and Developmental Biology, University of Michigan, Ann Arbor, MI, US.

\section{Differentiation into human definitive endoderm}

Routinely, cells were maintained in KO-DMEM supplemented with $10 \% \mathrm{KO}$ serum replacement, $1 \%$ non-essential amino acids, 1\% Glutamax, 0.1\% 2-mercaptoethanol, 1\% PEST (all from Gibco/ Invitrogen), $10 \%$ plasmanate (Talecris), and $10 \mathrm{ng} / \mathrm{mL}$ bFGF (Invitrogen). Medium was changed daily. Cells were passaged with $0.05 \%$ trypsin-EDTA (Gibco/Invitrogen) every third or fourth day onto freshly seeded $\left(25,000 / \mathrm{cm}^{2}\right)$ mitotically inactivated mouse embryonic feeder-cells (Sahlgrenska Academy Experimental Biomedicine University of Gothenburg, Gothenburg, Sweden). HUES3 was obtained from D.A. Melton, Howard Hughes Medical Institute (Harvard institute, Cambridge, MA, US). Human definitive endoderm was prepared as previously described by D' Amour and colleagues [21]. Briefly, a subclone of HUES-3 was seeded at a density of 20,000 cells/ $\mathrm{cm}^{2}$ on freshly seeded $\left(25,000\right.$ cells $\left./ \mathrm{cm}^{2}\right)$ mitotically inactivated MEFs. Cells were cultured according to protocols at http://mcb.harvard.edu/ melton/hues/ as previously described [22] for two to three days until a confluent monolayer was formed. Cells were washed once in PBS before the addition of differentiation medium. $100 \mathrm{ng} / \mathrm{mL}$ Activin A (R\&D systems) and $25 \mathrm{ng} / \mathrm{mL}$ Wingless-type MMTV integration site family, member 3a (Wnt3a; R\&D systems) was used to induce DE in Rosewell Park Memorial Institute (RPMI) 1640 (Gibco/Invitrogen) supplemented with no fetal bovine serum (FBS; Sigma) the first day and $0.2 \%$ FBS the following two days (Figure $3 \mathrm{~A}$ ). As a control for DEinduction, RPMI 1640 was used without addition of substances other than FBS. At day four, samples were taken for quantitative reverse transcription-PCR (qRT-PCR) analysis and immunocytochemical analysis. Bright field images of cells were taken on an inverted Eclipse TE2000-U microscope (Nikon).

\section{Immunocytochemical analysis of human and mouse definitive endoderm}

Cells were washed once with PBS and fixed for 15-30 minutes in $4 \%$ formaldehyde at room temperature followed by an additional PBS wash. Cells were permeabilized by washing in ethanol (70\%, 96\%, 99\%, $96 \%$, and $70 \%), 5$ minutes per step. Cells were rinsed $3 \times 5$ minutes in PBS followed by incubation with blocking buffer (10\% donkey serum (Jackson Immunoresearch) in PBS) for 30 minutes at room temperature. The cells were subsequently incubated with the primary antibody in blocking buffer over night at room temperature. The following primary antibodies were used: rat anti-Ecad (1:1000; Zymed Laboratories/Invitrogen) and goat anti-Foxa2 (1:200; Santa Cruz Biotechnology). Cell nuclei were counterstained using 4',6-diamidino2-phenylindole (DAPI; 1:2000). The following day, cells were washed thrice with PBS and then incubated for one hour at room temperature in the dark with the secondary antibody in blocking buffer, followed by three washes in PBS. The following secondary antibodies were used: Texas red-anti-rat (Invitrogen; 1:250) and Cy2-anti-goat (Jackson Immunoresearch; 1:250).

\section{mRNA extraction and reverse transcription}

Cells were harvested after trypsinization and total RNA was isolated with the GeneElute Mammalian Total RNA Miniprep kit (Sigma). RNA concentrations were determined by a NanoDrop ND-1000 spectrophotometer (Thermo Scientific). The reverse transcription was performed with SuperScript III (Invitrogen). Initially, 200 ng mRNA, 2 $\mu \mathrm{M}$ random hexamers, $2 \mu \mathrm{M}$ Oligo-dT primers (both from Invitrogen), and $10 \mathrm{mM}$ deoxynucleotidetriphosphates (dNTP; Fermentas) were incubated at $65^{\circ} \mathrm{C}$ for 5 minutes followed by cooling down to $8^{\circ} \mathrm{C}$. In the second step, $1 \times$ First Strand (FS) buffer, 5 mM DTT, 10 U Superscript $^{\mathrm{m}}$ III Reverse transcriptase, and 2 U RNaseOUT ${ }^{\mathrm{mx}}$ (all from Invitrogen), was added to a final reaction volume of $10 \mu \mathrm{L}$. The temperature profile was $25^{\circ} \mathrm{C}$ for 5 minutes, $50^{\circ} \mathrm{C}$ for 45 minutes, $55^{\circ} \mathrm{C}$ for 10 minutes, and $70^{\circ} \mathrm{C}$ for 15 minutes. Samples were diluted to $200 \mu \mathrm{L}$ with water and stored in $-20^{\circ} \mathrm{C}$ for later analysis by real-time PCR.

\section{Quantitative reverse transcription-polymerase chain reaction analysis}

Primers (Table 1) for qRT-PCR analysis were designed using Primer 3 (http://www-genome.wi.mit.edu/cgi-bin/primer/primer3 www.cgi). Reactions, using the 7900HT Fast Real-time PCR system (Applied biosystems), were performed accordingly: $400 \mathrm{nM}$ forward and reversed primers, 1x Platinum ${ }^{\circ}$ Quantitative PCR superMixUDG with ROX (Invitrogen), 0.125x SYBR green (Invitrogen), and $3 \mu \mathrm{L}$ template cDNA in $20 \mu \mathrm{L}$ reactions. The settings were used: Preincubation at $50^{\circ} \mathrm{C}$ for 2 minutes, and $95^{\circ} \mathrm{C}$ for 2 minutes followed by 45 cycles with denaturation at $95^{\circ} \mathrm{C}$ for $15 \mathrm{sec}$., annealing at $60^{\circ} \mathrm{C}$ for $25 \mathrm{sec}$., and extension at $73^{\circ} \mathrm{C}$ for $30 \mathrm{sec}$. Cycle of threshold (Ct)-values were determined using manual $\mathrm{Ct}$ and automatic baseline. The correct PCR-product was confirmed by agarose gel electrophoresis $(2 \% \mathrm{w} / \mathrm{v})$ and melting curve analysis. ACTB and Gapdh were verified as suitable house-keeping genes (HKGs) for $\mathrm{hDE}$ and $\mathrm{mDE}$ respectively. Relative quantification was calculated according to $1.9^{\wedge}(\mathrm{CtHKG}-\mathrm{Ct}$ gene $\mathrm{x})$, predicting an overall PCR efficiency of $90 \%$. The lowest value in each data set was arbitrarily set to one and the rest of the data points were related to this value. In each experiment, three biological replicates were measured. In addition, duplicate technical replicates were used throughout the measurements. Mean values \pm SEM were calculated.

\section{Preparation of chick embryos for grafting}

White leghorn chick embryos were cultured on an agar-albumen substrate using a filter paper carrier as described by Chapman et al [23]. Briefly, eggs were incubated at $38^{\circ} \mathrm{C}$ in a humidified incubator until the embryos reached Hamburger Hamilton (HH) stage 8-10 [24] After cracking, the egg contents were deposited in $10 \mathrm{~cm}$ Petri dishes with the embryos positioned at the top of the yolk (Figure 1A). The albumen covering the embryos was removed using pieces of filter paper (Figure 1B). A fresh piece of filter paper with a central aperture was then placed over each embryo and the vitelline membranes were cut around the circumference of the filter papers. The filter paper with the 


\begin{tabular}{|c|c|c|}
\hline Gene & Forward primer sequence & Reversed primer sequence \\
\hline H-ACTB & 5'-CTGGAACGGTGAAGGTGACA-3' & 5'- AAGGGACTTCCTGTAACAATGCA-3' \\
\hline H-FOXA2 & 5'-AGGAGGAAAACGGGAAAGAA-3' & 5'-CAACAACAGCAATGGAGGAG-3' \\
\hline $\mathrm{H}-\mathrm{CXCR} 4^{a}$ & 5'-CACCGCATCTGGAGAACCA-3' & 5'-GCCCATTTCCTCGGTCTAGTT-3' \\
\hline H-SOX17 & 5'-AAGGGCGAGTCCCGTATC-3' & 5'-TTGTAGTTGGGGTGGTCCTG-3' \\
\hline H-OCT4 & 5'-CGAAAGAGAAAGCGAACCAG-3' & 5'-AACCACACTCGGACCACATC-3' \\
\hline$H-G S C$ & 5'-GAGGAGAAAGTGGAGGTCTGG-3' & 5'- GCAAGAAAGTAGCATCGTCTG-3' \\
\hline$H-E C A D$ & 5'-CTGCCATTCTGGGGATTCT-3' & 5'- СTCTTCTCCGCCTCCTTCT-3' \\
\hline$H-N C A D$ & 5'-GGGTAATCCTCCCAAATCAAA-3' & 5'- CCACAAACATCAGCACAAGG-3' \\
\hline H-CER1 & 5'-CTTCTCAGGGGGTCATCTTG-3' & 5'- TCCCAAAGCAAAGGTTGTTC-3' \\
\hline$H-T R H$ & 5'-CTCTTCCTCCGGGAAAACAT-3' & 5'-TGGGTTACATCGACTGACCA-3' \\
\hline$H-M I X L 1^{a}$ & 5'-CTCTGACGCCGAGACTTGG-3' & 5'-CCAAGTCTCGGCGTCAGAG-3' \\
\hline$H-S O X 7$ & 5'-CACTGCTGAACTGGTCCCTAA-3' & 5'-TGGCTGGTGAGGAAGACAA-3' \\
\hline$H-T$ & 5'-CTACCCCAGCCCCTATGC-3' & 5'-CGTTGCTCACAGACCACAG-3' \\
\hline$H-P Y Y$ & 5'-CAGCGGTATGGGAAAAGAGA-3' & 5'-GAGTGCGTATGCAAATGACG-3' \\
\hline H-FGFR1 & 5'-GCCAGGACCCGAACAGAG-3' & 5'-CCCAGAAGAGGAGGCACTT-3' \\
\hline M-Sox17 & 5'-CTCGGGGATGTAAAGGTGAA-3' & 5'-GCTTCTCTGCCAAGGTCAAC-3' \\
\hline M-Foxa2 ${ }^{b}$ & 5'-TGGCTGCAGACACTTCCTACT-3' & 5'-CAACATCAGTACAACCCTCTGGT-3' \\
\hline M-Gapdh & 5'-CGGTGCTGAGTATGTCGTGGA-3' & 5'- GGCAGAAGGGGCGGAGATGA-3' \\
\hline M-Ecad & 5'-CGCTGAGATGGACAGAGAAG-3' & 5'-CAGGACCAGGAGAAGAGTGC-3' \\
\hline M-Ncad & 5'-CAGGTTTGGAATGGGTCTGT-3' & 5'-ATGTTGGGTGAAGGTGTGCT-3' \\
\hline M-Cer1 & 5'-AGGAGGAAGCCAAGAGGTTC-3' & 5'-CATTTGCCAAAGCAAAGGTT-3' \\
\hline M-Oct4 & 5'-CACGAGTGGAAAGCAACTCA-3' & 5'-AGATGGTGGTCTGGCTGAAC-3' \\
\hline$M-C x c r 4$ & 5'-ACGGCTGTAGAGCGAGTGTT-3' & 5'-CCGTCATGCTCCTTAGCTTC-3' \\
\hline$M-T r h$ & 5'-GCTGTGACTCCTGACCTTCC-3' & 5'-СССАААТСТССССТСТСТТC-3' \\
\hline M-Mixl1 & 5'-AGTTGCTGGAGCTCGTCTTC-3' & 5'-AGGGCAATGGAGGAAAACTC-3' \\
\hline M-Sox7 & 5'-GGATGAGAGGAAACGTCTGG-3' & 5'-GCTTGCCTTGTTTCTTCCTG-3' \\
\hline$M-T^{b}$ & 5'-AACTTTCCTCCATGTGCTGAGAC-3' & 5'-TGACTTCCCAACACAAAAAGCT-3' \\
\hline M-Pyy & 5'-GCAGCGGTATGGAAAAAGAG-3' & 5'-AACACACACAGCCCTCCAGT-3' \\
\hline M-Fgfr1 & 5'-CTGGACATCCCCAGAGAAAA-' & 5'-GAAGGCACCACAGAATCCAT-3' \\
\hline
\end{tabular}

$\mathrm{H}=$ primers for human genes, $M=$ primers for mouse genes. Primers marked with a or a ${ }^{\mathrm{b}}$ were designed according to $\mathrm{D}^{\prime} \mathrm{Amour}$ and colleagues ( $\mathrm{D}$ 'Amour et al., 2005; $\mathrm{D}$ 'Amour et al., 2006) or Tada and colleagues (Tada et al., 2005), respectively.

Table 1: Primer sequences used for quantitative RT-PCR.

adhering embryo was gently lifted from the yolk using forceps (Figure 1C). The embryo was gently washed in PBS and placed, ventral side up, in a $3.5 \mathrm{~cm}$ in diameter Petri dish containing an agar-albumen substratum (Figure 1D). The embryos were cultured in a humidified chamber at $38^{\circ} \mathrm{C}$ until grafting.

\section{Grafting of embryonic stem cell-derived endoderm to explanted chick embryos}

ESC-derived putative endoderm was stained with $10 \mu \mathrm{M}$ CellTracker $^{\text {Tix }}$ Orange CMTMR (5-(and -6)-((4-chloromethyl) benzoyl) amino) tetra-methylrhodamine; Molecular Probes/ Invitrogen) in cell culture media, for $30-60$ minutes at $37^{\circ} \mathrm{C}$. After labeling, the cells were washed twice in PBS and assembled into clumps of 10-50 cells by scraping. A small incision was made in the chick endoderm at the 4-8 somite pair position, using a sharpened tungsten needle. The cell clump was gently pushed down in the incision and positioned at the endoderm-mesoderm boundary. The cells were either placed laterally (Figures 1E,1F), or closer to the midline. After 48 hours incubation at $38^{\circ} \mathrm{C}$ in a humidified chamber, the chick embryos were dissected (Figures $1 \mathrm{G}, 1 \mathrm{H}$ ), fixed for 1 hour in $4 \%$ phosphate-buffered formaldehyde, $\mathrm{pH}$ 7.2, washed in PBS, and stored in absolute methanol $(\mathrm{MeOH})$ at $-20^{\circ} \mathrm{C}$.

\section{Whole mount immunofluorescence}

Grafted chick embryos were analyzed using whole mount immunofluorescence [25] to examine the integration of mouse DE
(mDE) or human DE (hDE) (Figure 2). Embryos were treated with Dent's bleach (MeOH:DMSO:H2O2, 4:1:1) [26] for two hours at room temperature followed by equilibration to PBS through a series of descending methanol concentrations in PBS (75\%,50\%, 25\%), 10 minutes each. The embryo was then treated with $0.5 \%$ TNB (a proprietary TSA-block supplied by Perkin-Elmer) for two hours, to block unspecific binding of the antibodies. Goat anti-Foxa2 (Santa Cruz Biotechnology) and/or mouse anti-Nkx6-1 (F55A10; Developmental Studies Hybridoma Bank/BCBC, University of Iowa, Iowa City, IA, US) was diluted $1: 1,000$ in $0.5 \%$ TNB and incubated overnight at $4^{\circ} \mathrm{C}$ with gentle rocking. The embryos were washed five times for one hour each with PBS. Cy2 conjugated secondary antibody (Jackson Immunoresearch) was diluted 1:500 in TNB and applied overnight at $4^{\circ} \mathrm{C}$ with gentle rocking. Finally, the embryos were washed three times for 20 minutes each with PBS.

\section{Sectioning, clearing, and mounting of grafted chick embryos}

Stained embryos were embedded in $4 \%$ agarose and $400 \mu \mathrm{m}$ transverse sections were obtained using a microtome (Vibratome). The collected sections were equilibrated to $100 \%$ methanol followed by optical clearing of the section for 10 minutes using BABB (1:2 mixture of benzyl alcohol to benzyl benzoate; both from Sigma-Aldrich). The equilibration to $\mathrm{MeOH}$ had to be complete to avoid any residual water in the tissue that would cause precipitation and render it opaque. The sections were mounted in BABB on objective slides and covered with cover slips prior to image capturing. 


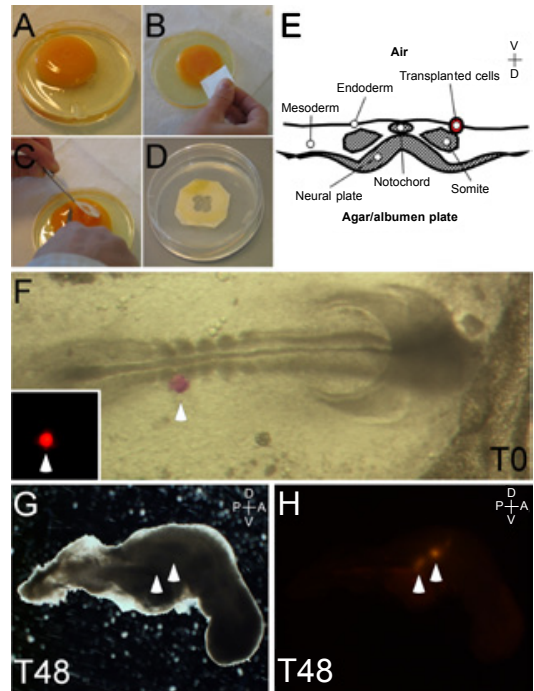

Figure 1: The method for grafting putative mouse and human definitive endoderm derived from embryonic stem cells to chick embryos. (A-D) The procedure of moving the chick embryo from the egg to the agar-albumen substrate using a filter paper carrier. (E) An illustration showing the positioning of grafted DE in the chick embryo, which is cultured ventral side up. $(F)$ Grafting of stained DE into the chick embryo. Inset: fluorescently labeled DE (CMTMR= CellTracker ${ }^{\mathrm{TM}}$ Orange dye). (G-H) Transplanted embryo 48 hours post grafting. The arrowheads point at sites of integration. $A=a n t e r i o r$, $\mathrm{D}=$ Dorsal, $\mathrm{P}=$ posterior, $\mathrm{V}=$ Ventral.

\section{Imaging}

All images of embryonic sections were obtained on a Zeiss LSM510 META Axio Imager connected to a LSM 510 laser module containing a $488 \mathrm{~nm}$ Argon laser, a $543 \mathrm{~nm}$ Helium-Neon laser, and a $633 \mathrm{~nm}$ Helium-Neon laser (Carl Zeiss). Pinholes for each channel were adjusted so that the optical section depths in each channel were equal. Emitted fluorescence was detected with line scans in multitrack mode using BP505 530-nm (Cy2). Fluorescence was recorded as square 8-bit images $(1024 \times 1024$ pixels $)$ and stored as separate image stacks for each channel. Images of immunofluorescently stained cells were taken on a Nikon Eclipse TE 2000-U Axioplan 2 fluorescence microscope equipped with ApoTome, and AxioVision LE software was used. Images were edited in Adobe ${ }^{\circledR}$ Photoshop version 8.0 or ACD Systems Canvas version 9.0.

\section{Results and Discussion}

In order to analyze the developmental potential of putative DE generated by directed differentiation of ESCs, we have established a transplantation assay. Briefly, chick embryos were explanted onto agar-albumen using a filter paper carrier [23] (Figures 1A-D), and incubated in a humidified chamber at $38^{\circ} \mathrm{C}$. Putative $\mathrm{mDE}$ or hDE was stained with the fluorescent dye CellTracker Orange CMTMR prior to transplantation, and subsequently transferred into the chick endoderm either in a lateral position (Figure $1 \mathrm{~F}$ ), or closer to the midline. The homing and integration of the transplanted cells was analyzed $48 \mathrm{~h}$ post grafting by whole mount immunofluorescence. This functional assay demonstrates integration of both putative hDE (Figures $2 \mathrm{~A}, 2 \mathrm{~B}$ ) and $\mathrm{mDE}$ (Figures 2C,2D) in the chick endoderm. Moreover, Sox17Hi mouse cells isolated from Activin A-treated Sox17GFP/+ mESCs, integrate in the Nkx6-1+ chick endoderm (Figures 2I-L) and can participate in the development of the pancreatic epithelium as previously reported [1]. Interestingly, control cells, not treated with Activin A, migrate away from the endoderm (Figures 2E-H), frequently homing towards an area adjacent to the neural tube ([1] and data not shown). This might reflect the high number of neuronal progenitors generated from ESCs in Activin A-deficient culture conditions. Although both $\mathrm{hDE}$ and $\mathrm{mDE}$ have integrated in the chick endoderm, there are differences in the frequency of integration ( $71 \% n=21$ and $27 \% n=26$ respectively). This could reflect intrinsic differences in the cell populations or species differences. Notably, the DE is not prepared according to the same protocols, but rather according to the DE protocols developed for each species. Activin A is added to hESC for three days onto a confluent monolayer of cells, whereas mESC are exposed to Activin A for five days, starting directly when the cells are seeded. However, the gene and protein expression data for the putative $\mathrm{hDE}$ and $\mathrm{mDE}$ are similar prior to transplantation. Both $\mathrm{hDE}$ and $\mathrm{mDE}$ express low levels of POU5F1 transcripts (encoding the pluripotency transcription factor OCT4), whereas the expression of key DE markers, including SOX17, FOXA2 (also known as HNF3B) and CXCR4 [27,28,29], are upregulated (Figure 3C). (N.B. The human gene nomenclature (http:// www.genenames.org) is used here although we refer to both human and mouse genes). This indicates endodermal character of both the human and mouse cell populations treated with Activin A. Notably, the majority of markers expressed in DE, including CER1, FOXA2, SOX17 and CXCR4, are also expressed in other germ layers [29-34]. Cerberus-like (Cer1), an antagonist of BMP and Nodal signaling [31], is first expressed in the anterior visceral endoderm (VE) of the embryo but later on also in the anterior DE [35]. We find that CER1 is upregulated in both putative hDE and $\mathrm{mDE}$ (Figure 3C). Thyrotropinreleasing hormone (Trh), here upregulated in both putative $\mathrm{mDE}$ and $\mathrm{hDE}$ (Figure 3C), transiently marks the entire DE population and is not expressed in extra embryonic endoderm [36]. However, Sox7, expressed in the mouse primitive, parietal and VE, but not in DE during early embryogenesis [28], is expressed in $\mathrm{hDE}$ and $\mathrm{mDE}$ cultures. The implication for further differentiation is unclear, as a recent study indicates that disperse VE cells participate in the development of the early gut tube [37]. To further evaluate the fate of the cultured cells, we have analyzed the expression of primitive streak (PS) markers. We can detect expression of the PS and nascent mesoderm marker MIXL1,

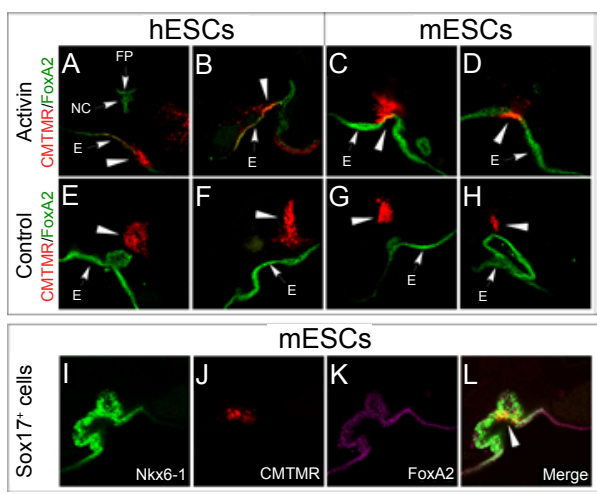

Figure 2: Sections of chick embryos 48 hours post grafting. The embryos were stained by whole-mount immunofluorescence followed by microtome sectioning. Activin A-induced putative DE derived from $h E S C$ (A-B) or mESCs $(C-D)$ integrate in the chick endoderm after grafting, whereas hESC progeny (E-F) or mESC progeny $(\mathrm{G}-\mathrm{H})$ not treated with Activin $A$ are rarely found in the developing endoderm. (I-L) Grafted Sox17+ mDE integrates in the chick endoderm and can participate in the development of the Nkx6-1+ chick pancreatic endoderm. The GFP signal from the Sox $17+$ cells is quenched during the whole mount staining procedure, which permit the detection of Nkx6-1 in the green channel. The arrowhead points at site of integration CMTMR $=$ CellTracker ${ }^{\mathrm{TM}}$ Orange dye, $\mathrm{FP}=$ Floor plate, $\mathrm{E}=$ Endoderm, NT= Neural tube. 

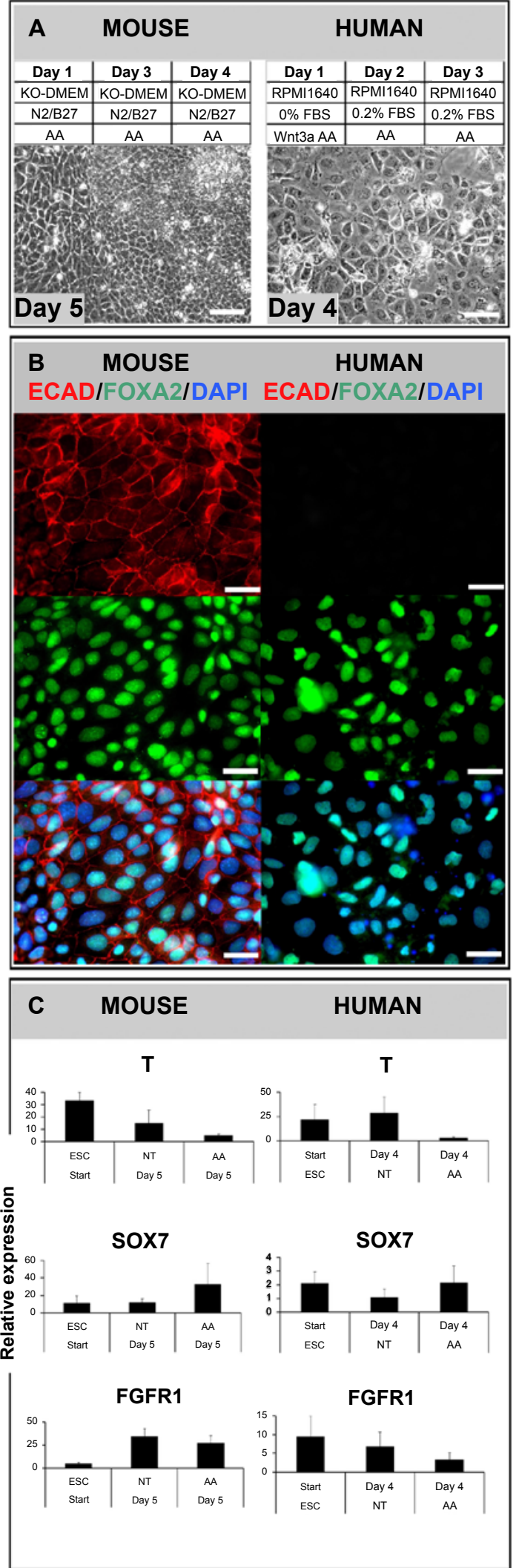

C MOUSE HUMAN

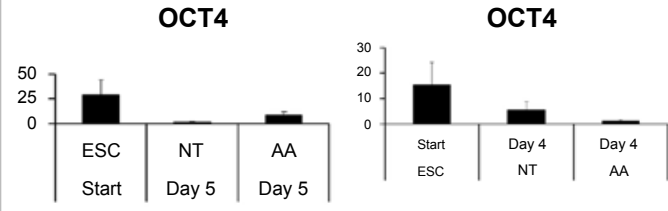

CXCR4

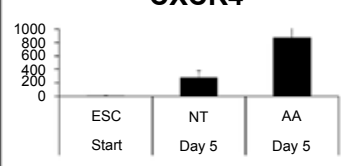

CXCR4

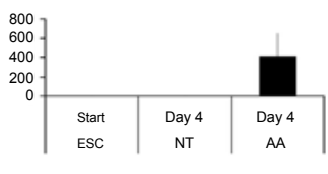

SoX17

SOX17

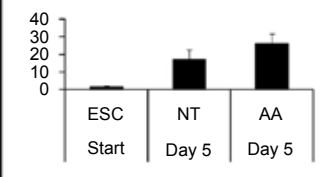

FOXA2
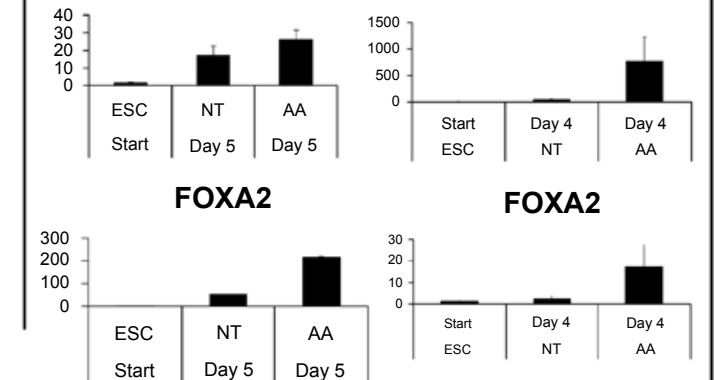

FOXA2

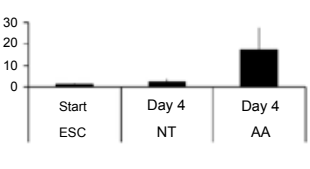

MIXL1
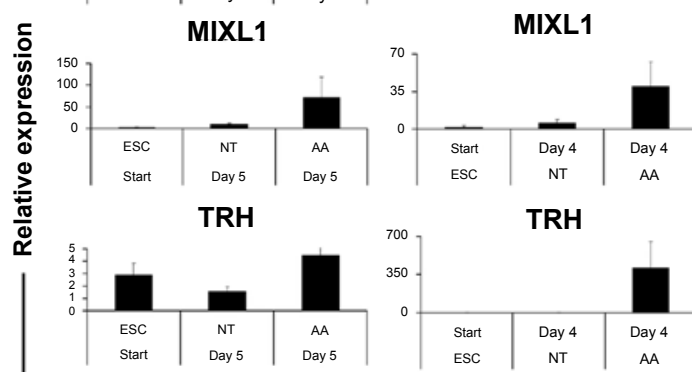

TRH

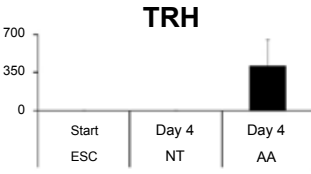

CER1

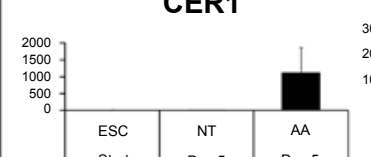

CER1

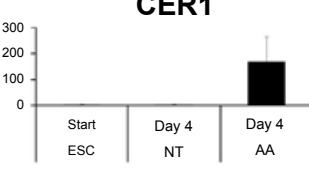

ECAD
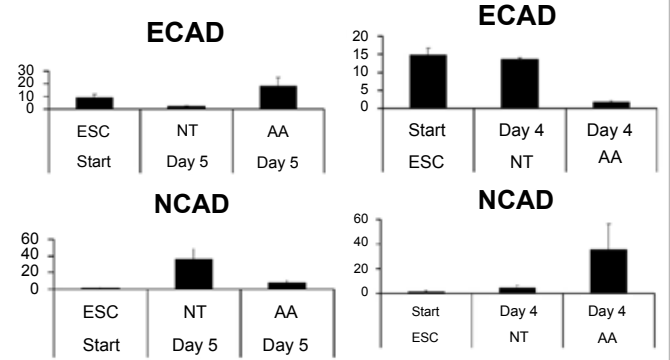

PYY

PYY

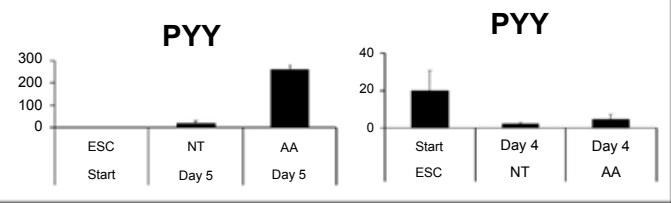

Figure 3: Analysis of putative human and mouse definitive endoderm prior to grafting. (A) The differentiation protocols for deriving putative mouse and human $D E$ from ESCs and bright-field images of mouse and human DE. Scale bars: $25 \mu \mathrm{m}$. (B) Immunofluorescence stainings (Sox17 and Ecad/FoxA2) of human and mouse DE. Nuclei are indicated by DAPI staining. Scale bars: $50 \mu \mathrm{m}$. (C) Relative mRNA-expression of genes in undifferentiated cells (ESC) as well as differentiated progeny treated with or without Activin A. NT = no treatment, AA = Activin A treatment. 
whereas the expression of T (BRACHYURY) is low, indicating that the cells have passed through the PS stage (Figure 3C).

One significant difference between the $\mathrm{hDE}$ and $\mathrm{mDE}$ is the expression of cadherins. mDE express E-cadherin (Cdh1; E-CAD in Figures 3B,3C) and not N-cadherin (Cdh2; N-CAD in Figure 3C), whereas the opposite is observed for hDE. E-cadherin is expressed throughout the epiblast and endoderm of the early embryo but is downregulated as epiblast cells undergo an epithelial-to-mesenchymal (EMT) transition at the PS [38]. Thus, we postulate that the putative $\mathrm{hDE}$ cells represent a late migratory mesendodermal population, rather than fully differentiated DE, which might also explain the lower frequency of integration. Furthermore, PYY, a novel endoderm marker [39], is upregulated in $\mathrm{mDE}$, whereas it is downregulated in $\mathrm{hDE}$ as well as in control cells (Figure 3C). Conclusively, the differentiation protocols for both hESC and mESC generate heterogeneous cell populations, but the resulting cells indeed represent populations of $\mathrm{DE}$.

The growth factor composition in the culture medium is slightly different for inducing $\mathrm{hDE}$ and $\mathrm{mDE}$. Wnt3a is added to the human cells in order to accelerate transition through the PS $[21,40]$ but not to the mESCs. The activation of Wnt signaling and induction of PS formation requires external signals but is self-reinforcing after initiation [40]. Wnt3a is coexpressed with Brachyury, which is a direct target of Wnt3a signaling [41] in the PS during gastrulation [42,43]. Furthermore, signaling through fibroblast growth factor receptor 1 (FGFR1) has been implicated in the EMT occurring at the PS by modulating E-cadherin and Wnt3a expression [44]. Determination of the expression profile of FGFR1 in ESC-derived DE reveals that FGFR1 transcripts are detected in $\mathrm{mDE}$ but has a low expression in $\mathrm{hDE}$. Nodal controls the formation of both endoderm and mesoderm in vivo $[45,46]$. However, in an attempt to recapitulate the in vivo developmental pathway, Activin $\mathrm{A}$, which has a higher bioactivity than Nodal, has been used as a substitute. It is believed to act in a similar manner since it has the same receptors as Nodal, apart from not having the co-receptor Cripto [47]. However, the use of Activin A could be an important difference between in vivo and in vitro formation of DE.

\section{Conclusions}

Importantly, the utility of ESC derivatives for further research and clinical applications critically depends on the ability to determine the functionality of the in vitro derived cells. The in vivo functionality of ESC-derived DE has previously been investigated in some studies using mouse or chick embryos $[19,48]$. Here, we demonstrate a detailed description of an in vivo functional assay for putative DE. Transplanted ESC-derived DE cells can integrate in the endoderm of the developing chick endoderm and participate in the generation of the gut tube and associated organs.

\section{Acknowledgement}

This work was supported by JDRF-Center for Beta cell Therapy in Europe (supported by JDRF \& EU 6th framework programme), Juvenile Diabetes Research Foundation (JDRF), Swedish Research Council, Swedish Diabetes Association Research Foundation, Stem Cell Center and Lund University. MJ was supported by the Medical Faculty of Lund University. We are grateful to D. A. Melton for providing hESC lines and S. Morrison for the Sox17GFP/+ mESC. We thank Anders Ståhlberg for designing various primer sequences. We thank Dr. Maria Harrie, AnnKatrin Häger, Karolina Landerman, Ingrid Sandelin, Jenny Ekberg, Ingar Nilsson, Morten Kahn, Maria Winzi and Ragna Jørgensen for technical assistance.

\section{References}

1. Shapiro AM, Ricordi C, Hering BJ, Auchincloss H, Lindblad R, et al. (2006) International trial of the Edmonton protocol for islet transplantation. $\mathrm{N} \mathrm{Engl} \mathrm{J}$ Med 355: 1318-1330.
2. Fiorina P Shapiro AM, Ricordi C, Secchi A (2008) The clinical impact of islet transplantation. Am J Transplant 8: 1990-1997.

3. Hogan A, Pileggi A, Ricordi C (2008) Transplantation: current developments and future directions; the future of clinical islet transplantation as a cure for diabetes. Front Biosci 13: 1192-1205.

4. Tam PP, Loebel DA (2007) Gene function in mouse embryogenesis: get set for gastrulation. Nat Rev Genet 8: 368-381

5. D'Amour KA, Bang AG, Eliazer S, Kelly OG, Agulnick AD, et al. (2006) Production of pancreatic hormone-expressing endocrine cells from human embryonic stem cells. Nat Biotechnol 24: 1392-1401.

6. Jiang J, Au M, Lu K, Eshpeter A, Korbutt G, et al. (2007) Generation of insulinproducing islet-like clusters from human embryonic stem cells. Stem Cells 25 1940-1953.

7. Phillips BW, Hentze H, Rust WL, Chen QP, Chipperfield H, et al. (2007) Directed differentiation of human embryonic stem cells into the pancreatic endocrine lineage. Stem Cells Dev 16: 561-578.

8. Kroon E, Martinson LA, Kadoya K, Bang AG, Kelly OG, et al. (2008) Pancreatic endoderm derived from human embryonic stem cells generates glucoseresponsive insulin-secreting cells in vivo. Nat Biotechnol 26: 443-452.

9. Semb H (2008) Definitive endoderm: a key step in coaxing human embryonic stem cells into transplantable beta-cells. Biochem Soc Trans 36: 272-275.

10. Stern CD (2005) The chick; a great model system becomes even greater. Dev Cell 8: 9-17.

11. Davey MG, Tickle C (2007) The chicken as a model for embryonic development. Cytogenet Genome Res 117: 231-239.

12. Wichterle H, Peljto M, Nedelec S (2009) Xenotransplantation of embryonic stem cell-derived motor neurons into the developing chick spinal cord. Methods Mol Biol 482: 171-183.

13. Lee H, Shamy GA, Elkabetz Y, Schofield CM, Harrsion NL, et al. (2007) Directed differentiation and transplantation of human embryonic stem cellderived motoneurons. Stem Cells 25: 1931-1939.

14. Motohashi T, Aoki H, Chiba K, Yoshimura N, Kunisada T (2007) Multipotent cell fate of neural crest-like cells derived from embryonic stem cells. Stem Cells 25: $402-410$

15. Yamashita J, Itoh H, Hirashima M, Ogawa M, Nishikawa S, et al. (2000) Flk1 positive cells derived from embryonic stem cells serve as vascular progenitors. Nature 408: 92-96.

16. Park TS, Zambidis ET, Lucitti JL, Logar A, Keller BB, et al. (2009) Human embryonic stem cell-derived hematoendothelial progenitors engraft chicken embryos. Exp Hematol 37: 31-41.

17. Goldstein RS, Drukker M, Reubinoff BE, Benvenisty N (2002) Integration and differentiation of human embryonic stem cells transplanted to the chick embryo. Dev Dyn 225: 80-86

18. Wichterle H, Lieberam I, Porter JA, Jessell TM (2002) Directed differentiation of embryonic stem cells into motor neurons. Cell 110: 385-397.

19. Hansson M, Olesen DR, Peterslund JM, Engberg N, Kahn M, et al. (2009) A late requirement for Wnt and FGF signaling during activin-induced formation of foregut endoderm from mouse embryonic stem cells. Dev Biol 330: 286-304.

20. Ying QL, Nichols J, Chambers I, Smith A (2003) BMP induction of Id proteins suppresses differentiation and sustains embryonic stem cell self-renewal in collaboration with STAT3. Cell 115: 281-292.

21. D'Amour KA, Agulnick AD, Eliazer S, Kelly OG, Kroon E, et al. (2005) Efficien differentiation of human embryonic stem cells to definitive endoderm. Nat Biotechnol 23: 1534-1541.

22. Cowan CA, Klimanskaya I, McMahon J, Atienza J, Witmyer J, et al. (2004 Derivation of embryonic stem-cell lines from human blastocysts. N Engl J Med 350: $1353-1356$

23. Chapman SC, Collignon J, Schoenwolf GC, Lumsden A (2001) Improved method for chick whole-embryo culture using a filter paper carrier. Dev Dyn 220: 284-289.

24. Hamburger V, Hamilton HL (1951) A series of normal stages in the development of the chick embryo. J Morphol 88: 49-92.

25. Ahnfelt-Rønne J, Jørgensen MC, Hald J, Madsen OD, Serup P, et al. (2007) 
Citation: Johannesson M, Semb H, Serup P, Hansson M (2012) A Functional Assay for Putative Mouse and Human Definitive Endoderm using Chick Whole-Embryo Cultures. J Stem Cell Res Ther 2:128. doi:10.4172/2157-7633.1000128

An improved method for three-dimensional reconstruction of protein expression patterns in intact mouse and chicken embryos and organs. J Histochem Cytochem 55: 925-930.

26. Dent JA, Polson AG, Klymkowsky MW (1989) A whole-moun immunocytochemical analysis of the expression of the intermediate filament protein vimentin in Xenopus. Development 105: 61-74.

27. D'Amour KA, Agulnick AD, Eliazer S, Kelly OG, Kroon E, et al. (2005) Efficient differentiation of human embryonic stem cells to definitive endoderm. Nat Biotechnol 23: 1534-1541.

28. McGrath KE, Koniski AD, Maltby KM, McGann JK, Palis J (1999) Embryonic expression and function of the chemokine SDF-1 and its receptor, CXCR4. Dev Biol 213: 442-456.

29. Kanai-Azuma M, Kanai Y, Gad JM, Tajima Y, Taya C, et al. (2002) Depletion of definitive gut endoderm in Sox17-null mutant mice. Development 129: $2367-$ 2379.

30. Ang SL, Wierda A, Wong D, Stevens KA, Cascio S, et al. (1993) The formation and maintenance of the definitive endoderm lineage in the mouse: involvement of HNF3/forkhead proteins. Development 119: 1301-1315.

31. Belo JA, Bachiller D, Agius E, Kemp C, Borges AC, et al. (2000) Cerberus-like is a secreted BMP and nodal antagonist not essential for mouse development. Genesis 26: 265-270.

32. Hallonet M, Kaestner KH, Martin-Parras L, Sasaki H, Betz UA, et al. (2002) Maintenance of the specification of the anterior definitive endoderm and forebrain depends on the axial mesendoderm: a study using HNF3beta/Foxa2 conditional mutants. Dev Biol 243: 20-33.

33. Yasunaga M, Tada S, Torikai-Nishikawa S, Nakano Y, Okada M, et al. (2005) Induction and monitoring of definitive and visceral endoderm differentiation of mouse ES cells. Nat Biotechnol 23: 1542-1550.

34. Sherwood RI, Jitianu C, Cleaver O, Shaywitz DA, Lamenzo JO, et al. (2007) Prospective isolation and global gene expression analysis of definitive and visceral endoderm. Dev Biol 304: 541-555.

35. Shawlot W, Deng JM, Behringer RR (1998) Expression of the mouse cerberus-related gene, Cerr1, suggests a role in anterior neural induction and somitogenesis. Proc Natl Acad Sci USA 95: 6198-6203.

36. McKnight KD, Hou J, Hoodless PA (2007) Dynamic expression of thyrotropinreleasing hormone in the mouse definitive endoderm. Dev Dyn 236: 2909-2917.
37. Kwon GS, Viotti M, Hadjantonakis AK (2008) The endoderm of the mouse embryo arises by dynamic widespread intercalation of embryonic and extraembryonic lineages. Dev Cell 15: 509-520.

38. Damjanov I, Damjanov A, Damsky CH (1986) Developmentally regulated expression of the cell-cell adhesion glycoprotein cell-CAM 120/80 in periimplantation mouse embryos and extraembryonic membranes. Dev Biol 116: 194-202.

39. Hou J, Charters AM, Lee SC, Zhao Y, Wu MK, et al. (2007) A systematic screen for genes expressed in definitive endoderm by Serial Analysis of Gene Expression (SAGE). BMC Dev Biol 7: 92.

40. ten Berge D, Koole W, Fuerer C, Fish M, Eroglu E, et al. (2008) Wnt signaling mediates self-organization and axis formation in embryoid bodies. Cell Stem Cell 3: 508-518.

41. Yamaguchi TP, Takada S, Yoshikawa Y, Wu N, McMahon AP (1999) T (Brachyury) is a direct target of Wnt3a during paraxial mesoderm specification. Genes Dev 13: 3185-3190.

42. Takada S, Stark KL, Shea MJ, Vassileva G, McMahon JA, et al. (1994) Wnt-3a regulates somite and tailbud formation in the mouse embryo. Genes Dev 8 : 174-189.

43. Chapman DL, Agulnik I, Hancock S, Silver LM, Papaioannou VE (1996) Tbx6, a mouse T-Box gene implicated in paraxial mesoderm formation at gastrulation. Dev Biol 180: 534-542.

44. Ciruna B, Rossant J (2001) FGF signaling regulates mesoderm cell fate specification and morphogenetic movement at the primitive streak. Dev Cell 1: $37-49$.

45. Grapin-Botton A Constam D (2007) Evolution of the mechanisms and molecular control of endoderm formation. Mech Dev 124: 253-278.

46. Zorn AM, Wells JM (2007) Molecular basis of vertebrate endoderm development. Int Rev Cytol 259: 49-111.

47. Kumar A, Novoselov V, Celeste AJ, Wolfman NM, ten Dijke P, et al. (2001) Nodal signaling uses activin and transforming growth factor-beta receptorregulated Smads. J Biol Chem 276: 656-661.

48. Borowiak M, Maehr R, Chen S, Chen AE, Tang W, et al. (2009) Small molecules efficiently direct endodermal differentiation of mouse and human embryonic stem cells. Cell Stem Cell 4: 348-358. 